\section{The relationship between caries in the primary dentition at 5 years of age and permanent dentition at 10 years of age - a longitudinal study}

\begin{abstract}
Skeie MS, Raadal M et al. Int J Paediatr Dent 2006; 16: 152-160
More than 2 surfaces experiencing caries in primary $2^{\text {nd }}$ molars at age 5 predicted high risk of caries at age 10 .

Caries in the primary dentition is related to caries in the permanent dentition. In this study, 217 5-year-olds were examined in clinics in Bergen, Norway and 186 of them were re-examined at the age of 10 yrs. From the 10 yr examinations, a risk group was defined on the basis of dentine caries experience on mesial of first molars or any incisors, or total DMFS for all caries $>1$ SD above the mean. This group was $24 \%$ of the sample, and had mean DMFS of 8.0.

No caries was found in $1 / 4$ of the children, and $71 \%$ of dentine caries was found in 17\% of children. In 57 children with no caries at baseline, $61 \%$ had caries in permanent teeth at age 10, but $81 \%$ with caries at baseline had permanent caries. Caries in primary second molars had an OR of 12.3 for predicting membership of the risk group. The authors suggest such caries should be the basis of advice to children and parents.
\end{abstract}

doi:10.1038/sj.bdj.4813896

\section{SOCIOLOGY; ONCOLOGY}

\section{The correlation between indices of} deprivation and health related quality of life in patients with oral and oropharyngeal squamous cell carcinoma

Wooley E, Magennis P etal. Br J Oral Maxillofac Surg 2006; 44: 177-186

Less deprived patients reported some improvement in quality of life ( $Q \circ L)$ after surgery.

Deprivation is related to incidence and outcome of cancer. In this study, 278 consecutive patients (mean age 62 yrs; 65\% male) who had received surgery for oral and oropharyngeal cancer were asked to complete QoL questionnaires at baseline, 6, 12 and 18 months after treatment, and between $2 / 3$ and 3/4 did so at each stage. Demographic and clinical data were available. At presentation, deprivation was not linked to the level of disease or to QoL. By 12 months, 17\% of patients had died, and by 24 months, $26 \%$.

Patients surviving at 18 months and living in the least deprived areas reported better post-operative QoL then those in more deprived areas. In the least deprived group, there was a trend for improvement of QoL from 6 to 18 months, following an initial fall after surgery. Unmarried patients, smokers and those drinking $40+$ units of alcohol per week were more likely to show no improvement from 6 to 18 months.

\section{DENTAL TRAUMA}

Traumatic intrusion of permanent teeth. Part 2. A clinical study of the effect of preinjury and injury factors, such as sex, age, stage of root development, tooth location, and extent of injury including number of intruded teeth on 140 intruded permanent teeth

Andreasen JO, Bakland LK et al. Dent Traumato/ 2006; 22: 90-98

\section{Younger patients had fewer complications.}

This is part of a study started in 1955 in Copenhagen. Over that time, 216 intruded teeth presented in 151 patients, but not all satisfied inclusion or follow-up criteria, leaving 140 teeth in 114 patients (mean age 8 yrs; range 6-67) with at least 1 yr of followup. Where possible, patients were followed up 5 yrs.

In 124 cases, pulps were necrotic and related mainly to more developed roots; in all 16 cases of live pulps, the canals were subsequently obliterated. In 67 cases showing progressive root resorption, 24 related to infection (15 were stopped by endodontics), 31 to ankylosis (transient in 6) and 12 to both factors. Subsequent marginal bone loss occurred in 45. Out of 28 teeth subsequently lost, root resorption accounted for 17 . The authors conclude that there was less risk of complication where root formation was incomplete, and that this might relate to softer surrounding bone.

doi:10.1038/sj.bdj.4813898

\section{DENTAL TRAUMA}

Traumatic intrusion of permanent teeth. Part 3. A clinical study of the effect of treatment variables such as treatment delay, method of repositioning, type of splint, length of splinting and antibiotics on 140 teeth

\section{Andreasen JO, Bakland LK et al. Dent Traumato/ 2006; 22: 99-111}

\section{If root formation is complete, spontaneous eruption may} occur, but in older patients, surgical or orthodontic extrusion is indicated.

This is a part of the study in the above abstract, and relates treatment to complications of pulp necrosis, root resorption and marginal bone loss.

Treatment delay had little effect on outcome. Teeth which were not repositioned had fewer complications, and total eruption occurred in 2-13 months. There were no differences in outcome between 3 different splints used after surgical repositioning, nor between orthodontic and surgical repositioning which were used above 17 yrs of age. Tooth survival was better in teeth with incomplete roots. The study showed no effect of dentine sealing, but the authors felt it reasonable to retain this procedure because only 17 cases were encountered. Antibiotic use had no effect on outcome. 